\title{
The clinical and cost effectiveness of a virtual fracture clinic service
}

\author{
AN INTERRUPTED TIME SERIES ANALYSIS AND \\ BEFORE-AND-AFTER COMPARISON
}

A. McKirdy,

A. M. Imbuldeniya

West Middlesex

University Hospital,

Twickenham Road,

Isleworth TW7 6AF,

United Kingdom
- A. McKirdy, MBChB, BSc (Hons), Clinical Fellow in Trauma and Orthopaedics,

a. M. Imbuldeniya,

MBBS, B Med Sci, MSc, DIC,

FRCS(Tr\&Orth), Consultant

Trauma \& Orthopaedic Surgeon,

Department of Trauma and

Orthopaedics, West Middlesex

University Hospital, Twickenham

Road, Isleworth TW7 6AF UK.

Correspondence should be sent to A. McKirdy;

email anne.mckirdy@nhs.net

doi: $10.1302 / 2046-3758.65 . B J R-$ 2017-0330.R1

Bone Joint Res 2017;6:259-269.

Received: 9 December 2016;

Accepted: 10 February 2017

\section{Objectives}

To assess the clinical and cost-effectiveness of a virtual fracture clinic (VFC) model, and supplement the literature regarding this service as recommended by The National Institute for Health and Care Excellence (NICE) and the British Orthopaedic Association (BOA).

Methods

This was a retrospective study including all patients $(17116)$ referred to fracture clinics in a London District General Hospital from May 2013 to April 2016, using hospital-level data. We used interrupted time series analysis with segmented regression, and direct before-and-after comparison, to study the impact of VFCs introduced in December 2014 on six clinical parameters and on local Clinical Commissioning Group (CCG) spend. Student's $t$-tests were used for direct comparison, whilst segmented regression was employed for projection analysis.

\section{Results}

There were statistically significant reductions in numbers of new patients seen face-toface $(140.4$, SD 39.6 versus 461.6 , SD $61.63, p<0.0001)$, days to first orthopaedic review (5.2, SD 0.66 versus 10.9 , SD $1.5, p<0.0001$ ), discharges ( 33.5 , SD 3.66 versus 129.2 , SD 7.36 , $p<0.0001$ ) and non-attendees (14.82, SD 1.48 versus 60.47 , SD $2.68, p<0.0001$ ), in addition to a statistically significant increase in number of patients seen within 72 -hours $(46.4 \%$ 3873 of 8345 versus $5.1 \% 447$ of $8771, p<0.0001)$. There was a non-significant increase in consultation time of 1 minute 9 seconds ( 14 minutes 53 seconds SD 106 seconds versus 13 minutes 44 seconds SD 128 seconds, $p=0.0878$ ). VFC saved the local CCG $£ 67385.67$ in the first year and is set to save $£ 129885.67$ annually thereafter.

\section{Conclusions}

We have shown VFCs are clinically and cost-effective, with improvement across several clinical performance parameters and substantial financial savings for CCGs. To our knowledge this is the largest study addressing clinical practice implications of VFCs in England, using robust methodology to adjust for pre-existing trends. Further studies are required to appreciate whether our results are reproducible with local variations in the VFC model and payment tariffs.

Cite this article: Bone Joint Res 2017;6:259-269.

Keywords: Virtual fracture clinic, Interrupted time-series, Orthopaedics

\section{Article focus}

- Are virtual fracture clinics clinically effective? Outcome measures: a) number of new patients attending face-to-face clinics, b) clinical time per face-to-face patient, c) days to first clinical orthopaedic review, d) percentage of patients assessed in fracture clinic within 72-hours, e) number of patients discharged from first face-to-face appointment (proxy for unnecessary referrals), and f) number of non-attendances at first face-to-face appointment.

- Are virtual fracture clinics cost-effective? Outcome measure: g) total annual cost to local CCGs for fracture clinic services. 
- Our hypothesis $\left(\mathrm{H}_{1}\right)$ was that VFCs were associated with a reduction in number of patients attending face-to-face clinic, waiting times, discharge and nonattendance figures and financial spend by the CCG, but an increase in clinical time per patient and percentage of patients seen within 72 hours. The null hypothesis $\left(\mathrm{H}_{0}\right)$ was thus that there was no change in any of the above outcomes following the intervention.

\section{Key messages}

We have shown VFCs to be clinically effective, with improvements across several key clinical performance parameters.

- Our results indicate substantial cost-savings for local CCGs by implementing VFCs, with a predicted annual saving of $£ 129885.67$. If this is reproducible nationally, VFCs may provide huge financial savings for the NHS.

- Further research is required to determine if our results are reproducible, and to examine the impact of VFCs on other outcomes such as patient satisfaction and adverse outcomes.

\section{Strengths and limitations}

- This is the largest study in the literature relating to VFCs in terms of patient numbers, and also covers the longest time-frame.

- It uses a robust methodological approach, as recommended by Cochrane for examining the effects of such healthcare interventions.

- Limitations on available data pre-VFC meant reliably assessing patient satisfaction was outside the scope of this study.

\section{Introduction}

Orthopaedic conditions requiring outpatient fracture clinic follow-up are common. In England 1.8 million fractures occur annually with a lifetime prevalence of nearly $40 \%,{ }^{1}$ whilst $4.6 \%$ of all Emergency Department (ED) attendances are represented by dislocations, joint injuries, fractures and amputations. ${ }^{2}$

In the context of increasing ED attendances, 3,4 and a rising proportion of patients being assessed by staff with no formal orthopaedic training, many orthopaedic departments in the United Kingdom face increasingly overstretched fracture clinics where demand often exceeds capacity. This leads to prolonged waiting times; in many cases patients now wait up to two weeks. Such delays can potentially cause patient harm, particularly if suboptimal management plans were initiated on first presentation. Furthermore, many non-complex injuries heal with minimal intervention, yet with current models of working unnecessary time and expertise is often expended on these.

In August 2013 the British Orthopaedic Association (BOA) published guidelines for the standard of care patients should expect following injuries requiring fracture clinic follow-up, known as the British Orthopaedic Association Standards of Trauma (BOAST) 7 guidelines. ${ }^{5}$ The most challenging target for NHS Trusts to achieve given the above context was: "Following acute traumatic orthopaedic injury, patients should be seen in a new fracture clinic within 72 hours of presentation. This includes referrals from emergency departments, minor injury units and general practice."

'Virtual' fracture clinics (VFCs) have been developed in response to overstretched traditional fracture clinic models in the context of financial limitation, ${ }^{6,7}$ and a desire by clinicians to meet the BOAST 7 target. These clinics follow on from successful virtual clinics in other specialties. ${ }^{8-10}$ Currently 32 units in the United Kingdom have a fully implemented VFC model, ${ }^{11}$ the most notable was established in the Glasgow Royal Infirmary. The 'Glasgow' model has become the benchmark for NHS Trusts considering fracture clinic redesign, and their results have been widely published. ${ }^{12-17}$

Despite an increasing uptake of VFCs, there is a paucity of publications from other units. The BOA published a statement on their website in 2015 "welcoming research into all aspects of improving patient care and encouraging publication of the results of such trials of new fracture clinic models." 18 Additionally, the National Institute for Health and Care Excellence (NICE) in 2016 highlighted the importance of research comparing VFC to traditional fracture clinics - they concluded there was a need to understand "the clinical and cost-effectiveness of virtual new patient fracture clinics compared with consultant-led face-toface clinics in people presenting with non-complex fractures." 1

\section{Patients and Methods}

This retrospective interrupted time series (ITS) study comprised all patients referred to outpatient fracture clinics at a West London District General Hospital from May 2013 to April 2016 inclusive, including referrals from ED, Minor Injury Units (MIU) and GP. No patients were excluded.

Advice regarding the need for ethical approval was sought, however as pre-existing anonymised data was used this was not required.

Data collection: clinical (outcomes a-f). Data regarding outpatient activity including attendances, discharges, non-attendances and time to first clinic appointment are generated directly from the study site's electronic Patient Administrative System (PAS) and stored in the Trust's Outpatient Commissioning Data Set, which is used primarily for monitoring and payment purposes. Relevant data was received from our Information Governance Team in the form of Excel pivot-tables (Microsoft Corporation, Albuquerque, New Mexico), and included 
Table I. Comparison of National Tariff and locally-agreed tariff prices for each fracture clinic type

\begin{tabular}{lll}
\hline Clinic & National Tariff & $\begin{array}{l}\text { Local tariff for } \\
\text { study site }\end{array}$ \\
\hline New face-to-face clinic & $£ 129$ & $£ 160$ \\
Follow-up face-to-face clinic & $£ 77$ & $£ 96.08$ \\
Virtual clinic & $£ 27$ & $£ 64.03$ \\
\hline
\end{tabular}

monthly activity for all fracture clinics over the study period. No demographic data was available.

Data-collection: financial (outcome g). National Tariff prices for the different types of fracture clinics were obtained from various NHS England websites, ${ }^{19,20}$ whilst corresponding locally-agreed prices were verified by the Income and Contracts Manager within the Finance Team (Table I). In addition to agreeing higher tariff prices for all fracture clinic types (Table I), managers at the study site agreed with the CCG for the higher amount of $£ 160$ to be paid for all first follow-up appointments where patients were referred directly from VFC, in order to offset the anticipated drop in income resulting from fewer new face-to-face clinic referrals.

The Band 6 VFC Nurse salary was confirmed by our organisation's Human Resources team to be $£ 34530$ per annum in line with the national NHS pay scale, with additional London weighting giving a total of $£ 39013$ per annum. ${ }^{21}$

Our VFC model. The 'intervention' was the introduction of VFCs on 1 December 2014, in response to a transformation Commissioning for Quality and Innovation (CQUIN) target from the local CCG.

CCGs are local NHS organisations, created by the Health and Social Care Act (2012). As more than $90 \%$ of patients' NHS contact is with their GP, CCGs were created to enable GPs to buy the services that their patients need. All GP practices within a given geographical area work together as a CCG to buy services that they refer their patients on to, such as hospital, mental health and community services. CCGs are led by a governing body of elected local GPs supported by other clinicians and NHS managers.

The CQUIN scheme is a form of pay-for-performance, enabling CCGs to reward excellence by linking a proportion of healthcare providers' income to the achievement of local quality improvement goals.

Ours is a lean VFC model, requiring the employment of one new full-time Band 6 VFC Nurse but with no additional staffing or overhead costs. VFCs run alongside traditional

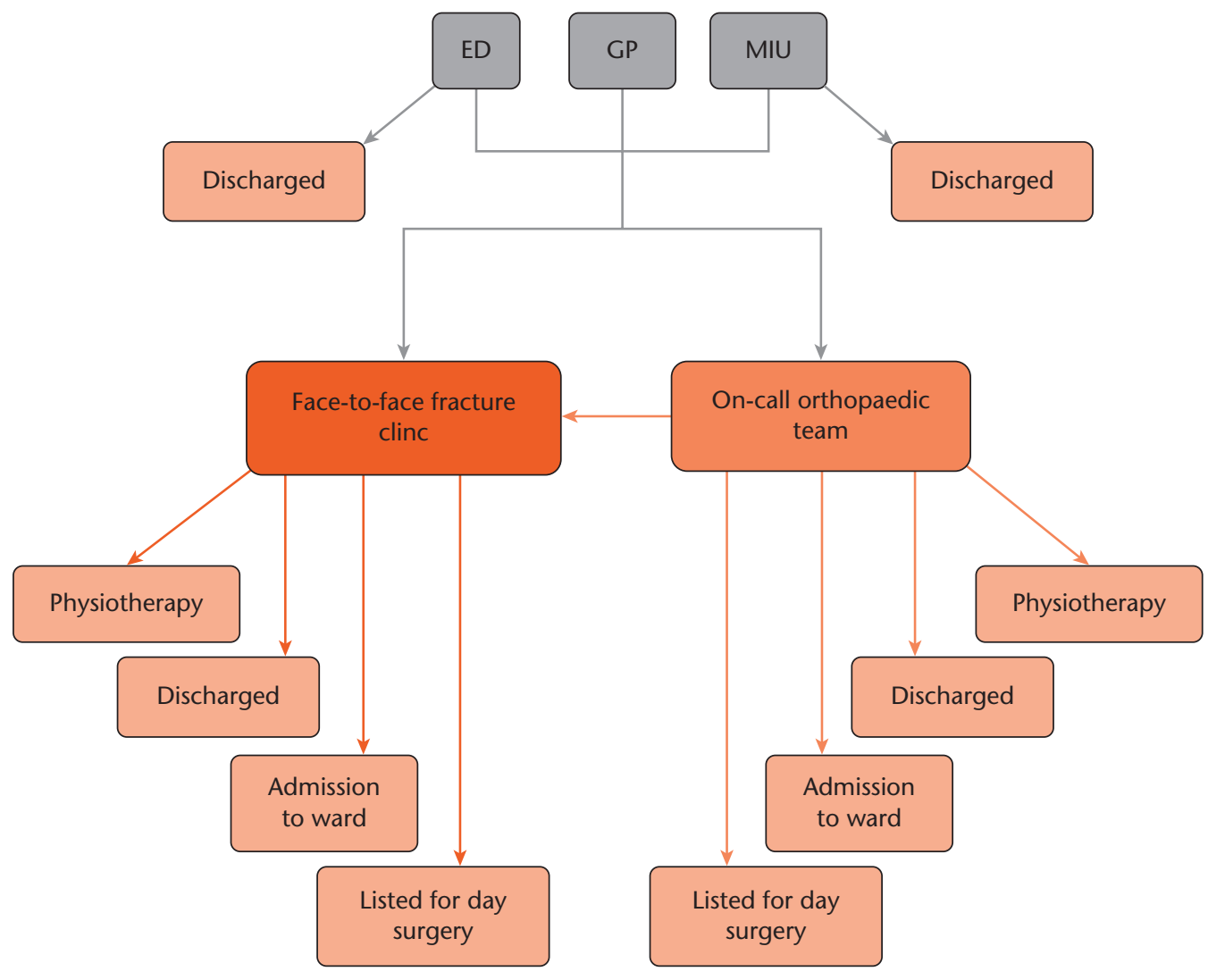

Fig. 1

Referral pathways to fracture clinics before the intervention. Emergency department (ED), GPs and minor injuries units (MIU) could directly book patients into face-to-face clinics in addition to the on-call orthopaedic team. 


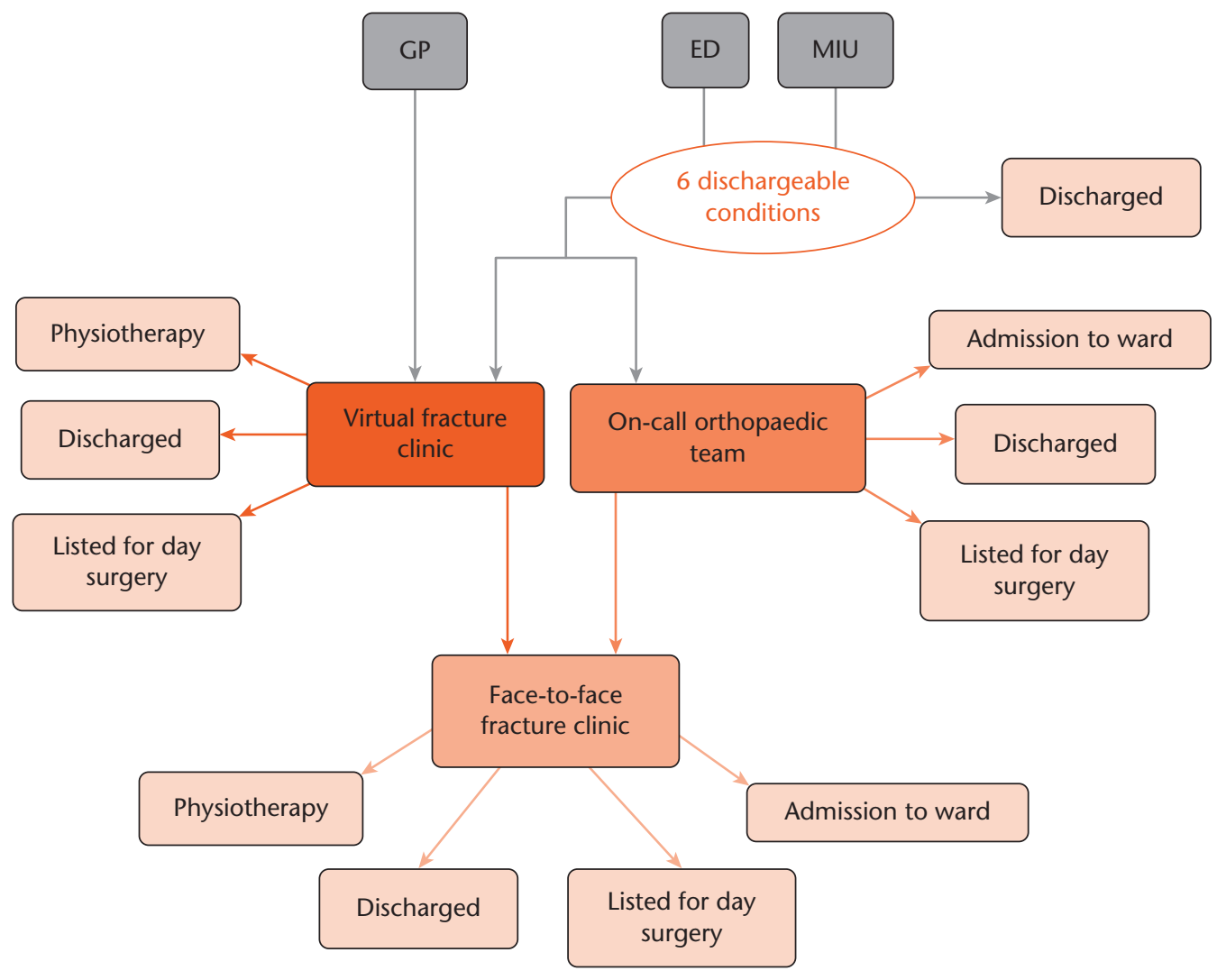

Fig. 2

Referral pathways to fracture clinics after the intervention. Emergency department (ED), GPs and minor injuries units (MIU) can book patients directly to VFC only, or refer to the on-call orthopaedic team which is the only route for booking into face-to-face clinic. Patients reviewed in VFC can subsequently be followedup in face-to-face clinics.

Table II. Figures and calculations used to determine the mean maximum clinical time per patient in face-to-face clinic in the pre- and post-intervention periods

\begin{tabular}{|c|c|c|}
\hline & Pre-intervention period & Post-intervention period \\
\hline Fracture clinics in whole period (n) & $\begin{array}{l}6 \text { clinics per week } \times 82 \text { weeks in pre-intervention } \\
\text { period }=492+3 \text { extra days in pre-intervention } \\
\text { periods }=495-11 \text { Bank Holidays }=484\end{array}$ & $\begin{array}{l}6 \text { clinics per week } \times 74 \text { weeks in post-intervention } \\
\text { period }=444-12 \text { Bank Holidays }=432\end{array}$ \\
\hline Mean number of fracture clinics per month & $484 / 19$ months in pre-intervention period $=25.47$ & 432,17 months in pre-intervention period $=25.41$ \\
\hline Total number of 'clinician-minutes' per clinic & 3 clinicians $\times 180$ minutes $=540$ & $\begin{array}{l}\text { ( } 2 \text { clinicians } \times 180 \text { minutes })+(1 \text { clinician } \times 90 \text { minutes } \\
=450\end{array}$ \\
\hline $\begin{array}{l}\text { Mean number of 'clinician minutes' per } \\
\text { month }\end{array}$ & $\begin{array}{l}540 \text { clinician-minutes per clinic } \times 25.47 \text { clinics per } \\
\text { month }=13753.8\end{array}$ & $\begin{array}{l}450 \text { clinician-minutes per clinic } \times 25.41 \text { clinics per } \\
\text { month }=11434.5\end{array}$ \\
\hline $\begin{array}{l}\text { Mean maximum 'clinician-minutes' per } \\
\text { patient }\end{array}$ & $\begin{array}{l}13753.8 \div \text { number of face-to-face attendances each } \\
\text { month }\end{array}$ & $\begin{array}{l}11434.5 \div \text { number of face-to-face attendances each } \\
\text { month }\end{array}$ \\
\hline
\end{tabular}

face-to-face clinics, with the consultant spending the first 90 minutes in VFC before joining his colleagues in face-toface clinic. Management plans are communicated directly to the patient via telephone thereafter by the VFC Nurse.

Referral pathways and patient flow through fracture clinics at the study site are shown both pre-intervention (Fig. 1) and post-intervention (Fig. 2). Access to face-toface clinic has been restricted to the on-call orthopaedic team, whilst ED, GP and MIU staff can book directly into VFC.

Study design: interrupted time series (outcome measures $\mathbf{a}, \mathbf{c}, \mathbf{e}, \mathbf{f})$. A useful tool for evaluating the effectiveness of healthcare interventions is ITS. ${ }^{22,23}$ Data is collected at several time-points both before and after a discrete intervention, to determine whether observed changes in outcome are explained by secular trends in the data or are likely attributable to the intervention itself. ${ }^{22}$ It also allows for controlling for confounders such as seasonality, ${ }^{23}$ and is preferable to case-control or cohort studies where the inclusion of a control group is unfeasible (such as in this instance where the whole population was subject to the new referral pathway). ${ }^{24}$

Our data meets the requirements for ITS analysis as set out by Wagner et $\mathrm{al}^{23}$ in 2002 , with clearly defined pre 


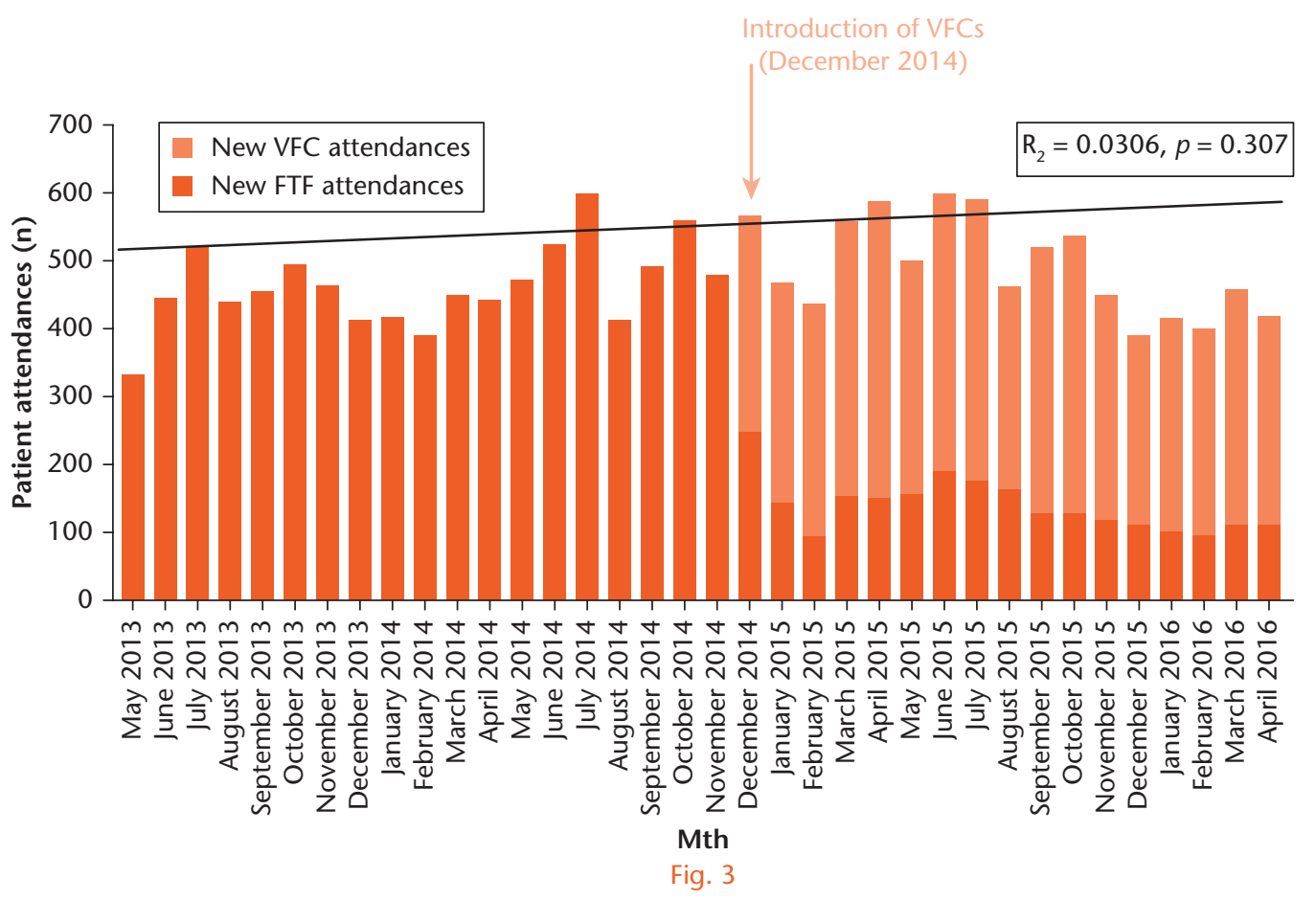

All new referrals to outpatient fracture clinics over the study period. Face-to-face (FTF) attendances are shown in dark orange whilst virtual fracture clinic (VFC) appointments are in pale orange. The solid black line indicates the trend line for total number of new referrals, which is a non-significant increase over time.

and post-intervention periods, similar numbers of patients in both groups $\left(\mathrm{n}_{1}=8771, \mathrm{n}_{2}=8345\right)$ and a continuous, repeated series of observations performed on the study population collected at 36 equal time-intervals (19 pre-intervention and 17 post-intervention).

Initially, summary and descriptive statistics were obtained to familiarise researchers with the data and to allow for a priori determination of a best-fit regression model - a level-and-slope-change model was chosen for all outcomes as follows: $Y_{t}=\beta_{0}+\beta_{1} T+\beta_{2} X_{t}+\beta_{3} T X_{t}$ (where $\mathrm{T}=$ time since start of the study period (months), $\mathrm{X}_{\mathrm{t}}=$ binary variable indicating pre-intervention (0) or postintervention (1) period, $Y_{t}=$ outcome at time $t, \beta_{0}=$ baseline level at $\mathrm{T}=0, \beta_{1}=$ pre-intervention trend or slope, $\beta_{2}=$ level-change following intervention and $\beta_{3}$ = slope change following intervention).

Segmented regression analysis with least-squares (ordinary) fit was performed. The node between pre- and post-intervention segments was constrained to datapoint 20 (December 2014; introduction of VFCs).

The presence of autocorrelation within the data was excluded by plotting the residuals and checking for normality (D'Agostino \& Pearson test); 25,26 autocorrelations were thus not modelled. There was minimal seasonality upon preliminary analysis therefore no dummy variables were included in the regression model.

During the study period there were no additional changes to the format of fracture clinic services, therefore we have made the assumption that VFC was the sole intervention for this population. No patient-specific covariates could be identified as no demographic data were available.

Before-and-after comparisons. For outcome measures $b$ and d we opted to perform simple before-and-after comparisons as data were non-continuous. Data was checked for normality using the D'Agostino \& Pearson test ${ }^{25,26}$ and parametric analyses performed appropriately.

Mean maximum clinician-time per patient in face-toface clinic was calculated using monthly attendance figures (obtained from the Trust data-set) and information regarding the number, duration and staffing of these clinics, for both pre- and post-intervention periods. Number of clinics: six face-to-face clinics per week, every week, however they do not run on Bank Holidays of which there are eight per calendar year. Clinic duration: three hours (180 minutes). Staffing: three orthopaedic surgeons. Since the introduction of VFC the consultant spends 90 minutes in VFC before joining his colleagues in face-to-face clinic. The total number of face-to-face 'clinician-minutes' per clinic was therefore 540 minutes in the pre-intervention period and 450 minutes thereafter.

Calculations are shown in Table II Mean clinician-time per patient was calculated by dividing by the mean number of clinician-minutes per month by the actual number of patients attending face-to-face for that particular month; monthly figures were used to allow more statistically accurate comparisons to be made between pre and post-intervention periods.

Financial (outcome measure $\boldsymbol{g}$ ). Before-and-after comparison of the cost to the CCG for commissioning all 


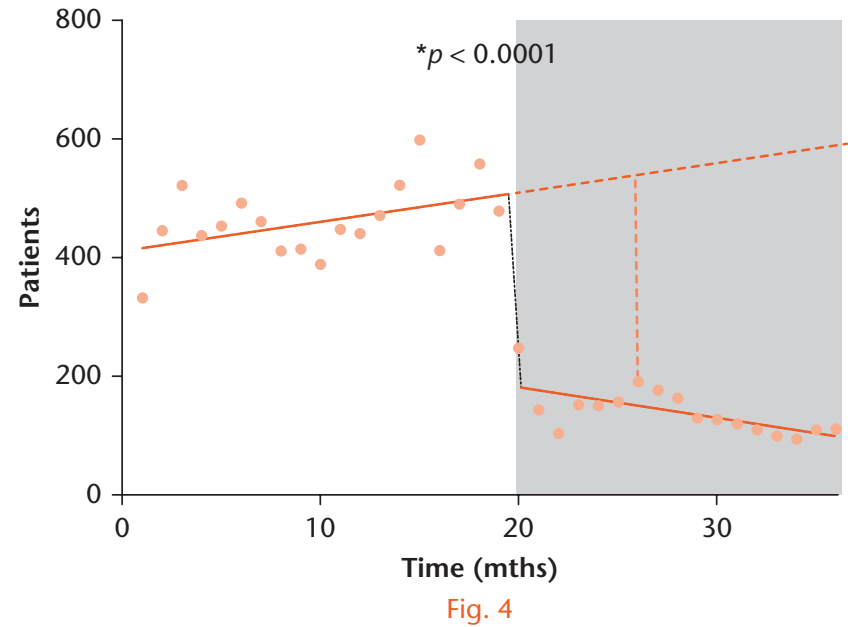

Interrupted time series (ITS) segmented regression for number of new patients seen in face-to-face fracture clinic per month, showing a level-andslope change at 20 months (December 2014, the intervention). Solid dark orange line, regression line for each segment; Dashed dark orange line, counterfactual scenario; light orange dotted line, difference between counterfactual and actual scenario at six months post-intervention; Grey shading, post-intervention period.

outpatient fracture clinic services at the study site was made for the financial year prior to the intervention and the financial year after. This was achieved by simple numerical calculations based on the attendance figures for each clinic type (obtained in the Trust data-set) and the agreed tariff prices (Table I).

Statistical analyses. Data was stored in Microsoft Excel workbooks (version 15.20, Microsoft Corporation), and all numerical calculations performed using programmed formulae available in this software. Statistical analyses were performed in Graph Pad Prism version 7.01 (La Jolla, San Diego California).

Student's $t$-test was the parametric test used for direct before-and-after comparisons as all data were found to be normally distributed, whilst segmented regression was performed to complete the ITS. All p-values reported are two-tailed, with an arbitrarilyassigned significance level of 0.05. All means are reported with standard deviation (SD).

\section{Results}

A total of 17116 new patients were reviewed in outpatient fracture clinics over the study period, 11158 in faceto-face and 5958 in VFC. Figure 3 illustrates this, with an overall increase in total number of new referrals per month (solid black line), although this was not statistically significant (linear regression, $p=0.307, R^{2}=0.0306$ ). Post-intervention, $71.4 \%$ (5958 of 8345) of all new fracture clinic appointments have been virtual.

Are VFCs clinically effective?: Number of new patients attending face-to-face clinic per month. ITS segmented regression for number of new patients seen in faceto-face clinic per month is shown in Figure 4. The

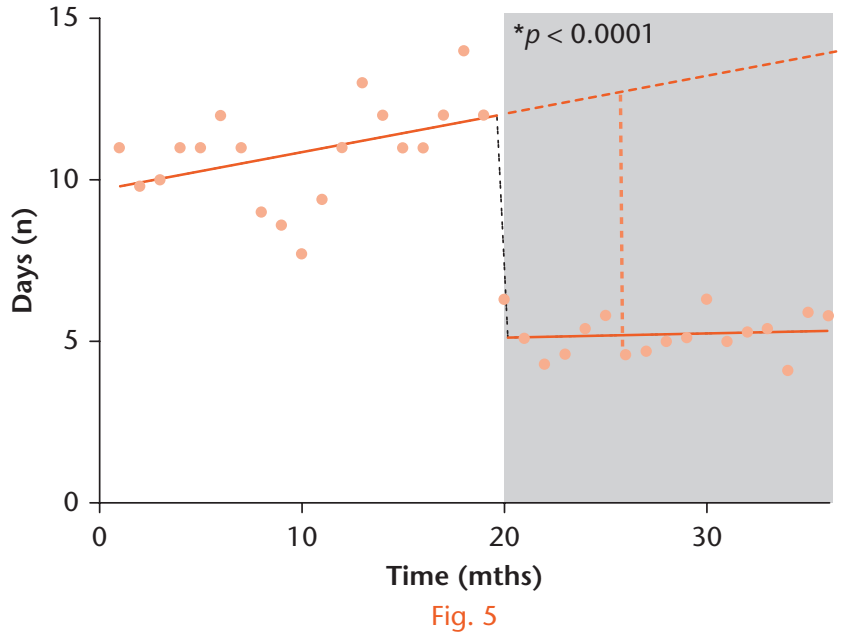

Interrupted time series (ITS) segmented regression for number of days to first clinical review by an orthopaedic doctor, showing a level-and-slope change at 20 months (December 2014, the intervention). Pre-intervention slope, 0.12; post-intervention slope, 0.013 . Solid dark orange line, regression line for each segment; Dashed dark orange line, counterfactual scenario; light orange dotted line, difference between counterfactual and actual scenario at six months post-intervention; Grey shading, post-intervention period.

post-intervention period is shaded grey, and the counterfactual scenario (expected scenario had the intervention not occurred, based on pre-existing data-trends) is indicated by a dashed line.

Pre-intervention, a mean 461.6, SD 61.63 patients were seen in face-to-face clinic per month with an upward trend indicating 4.9 additional patients seen month-onmonth. Post-intervention, there is a clear change in both slope and level with a mean 140.4, SD 39.62 patients seen and a downward trend indicating 5.2 fewer patients seen each month compared with the previous one. At six months after intervention, there is a statistically significant decrease in the number of patients seen in face-toface clinic of $27.8 \%$ compared to the counterfactual scenario (149.8 versus 539.4 patients per month, segmented regression $p<0.0001$ ).

Clinical time per patient in face-to-face clinics. Preintervention, each patient saw a doctor for a mean maximum time of 13 minutes 44 seconds (SD 2 minutes 8 seconds) increasing post-intervention to 14 minutes 53 seconds (SD 1 minute 46 seconds), a statistically nonsignificant increase of one minute nine seconds (Student's $t$-test, $p=0.0878$ ).

Number of days to orthopaedic clinical review. ITS segmented regression for patient wait between referral and first orthopaedic review in clinic is shown in Figure 5. Pre-intervention, the mean wait was 10.9, SD 1.5 days compared with 5.2, SD 0.66 days post-intervention (Student's $t$-test, $\mathrm{p}<0.0001$ ). The pre-intervention trend showed increasing wait-times, with a month-onmonth increase of 0.1 days. This trend slowed postintervention, now displaying a monthly increase of only 0.013 days. 


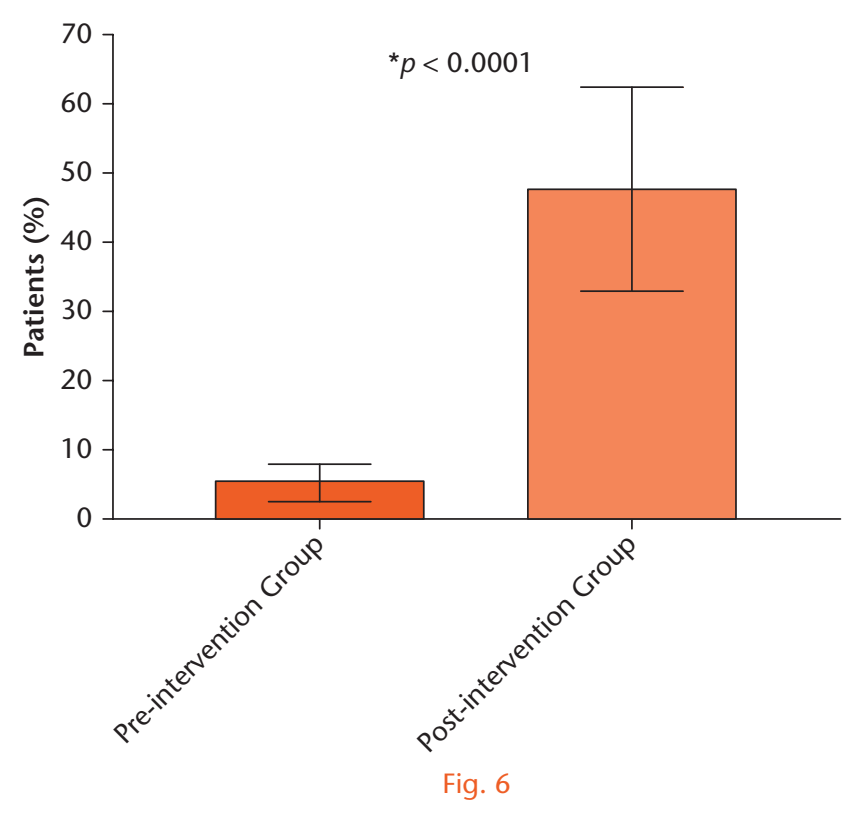

Graph of Student's t-test results comparing the percentage of patients reviewed within the BOAST 7 72-hour target time-frame in the postintervention vs pre-intervention groups. There was a significant increase from $5.1 \%( \pm 0.49)$ to $46.4 \%( \pm 3.29)$, Student's $t$-test, $p<0.0001$.

At six months post-intervention, the waiting time was 5.1 days but would have been expected to be 12.8 without the intervention; this is a significant decrease by 40.1\% (segmented regression, $\mathrm{p}<0.0001$ ).

Percentage of patients assessed within the 72-hour target recommended in BOAST 7 guidelines. Pre-intervention, $5.1 \%$ (SD $0.49 ; 447$ of 8771 ) of new patients referred to the fracture clinic had their first appointment within
72 hours, compared to a post-intervention figure of $46.4 \%$ (SD 3.29; 3873 of 8345 ); this is a statistically significant increase (Student's $t$-test, $p<0.0001$, Fig. 6).

Number of patients discharged from first face-to-face appointment. Monthly discharge rates from VFC since its introduction are shown in Figure 7; mean discharge rate is $32.9 \%$, ranging from $25.9 \%$ (January 2015 ) to $39.5 \%$ (April 2016).

Figure 8 shows ITS segmented regression for monthly numbers of patients discharged from first face-to-face fracture clinic, which acts as a proxy for unnecessary referrals as patients did not require further orthopaedic input.

Mean number of direct discharges decreased significantly from 129.2 (SD 7.36) to 33.5 (SD 3.66) per month following the intervention ( $p<0.0001$, Student's $t$-test). Pre-intervention there was an upward trend, with an increase of 3.7 discharges per month. Post-intervention, this trend has reversed with 1.5 fewer discharges monthon-month. At six months post-intervention, we would expect 188.2 direct discharges according to pre-intervention trends, therefore the observed figure of 38 discharges represents a $79.8 \%$ significant decrease (segmented regression, $\mathrm{p}<0.0001$ ).

Number of non-attendances at first face-to-face appointment. The mean absolute number of patients who did not attend their first face-to-face clinic appointment decreased from 60.5 (SD 2.68) to 14.8 (SD 1.48) per month after the intervention $(p<0.0001$; Student's $t$-test; Fig. 9). At six months post-intervention, there was a statistically significant $75.0 \%$ reduction in non-attendance figures between the observed values and those expected

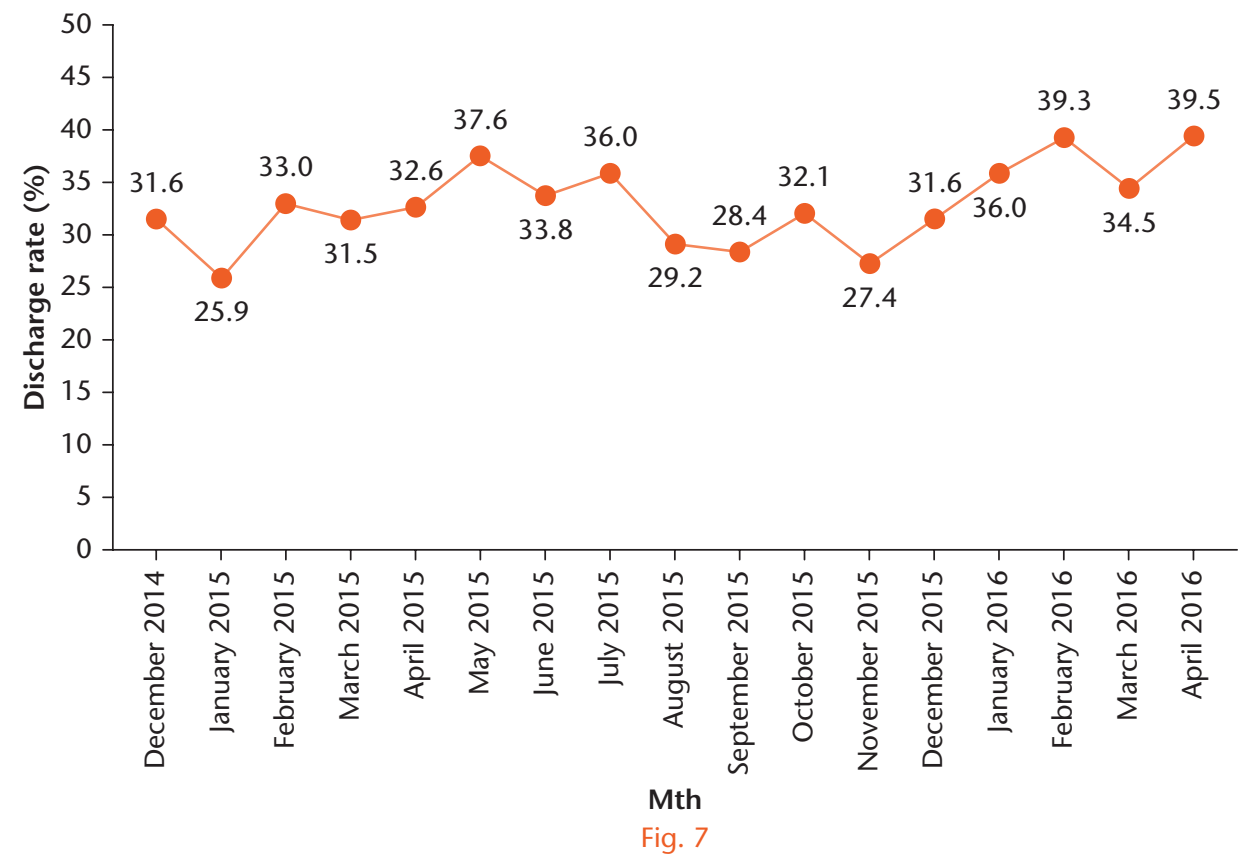

Graph of study site Virtual fracture clinic (VFC) discharge rates per month over the entire study period. Mean discharge rate is $32.9 \%$ (range 25.9 to $39.5 \%$ ). 


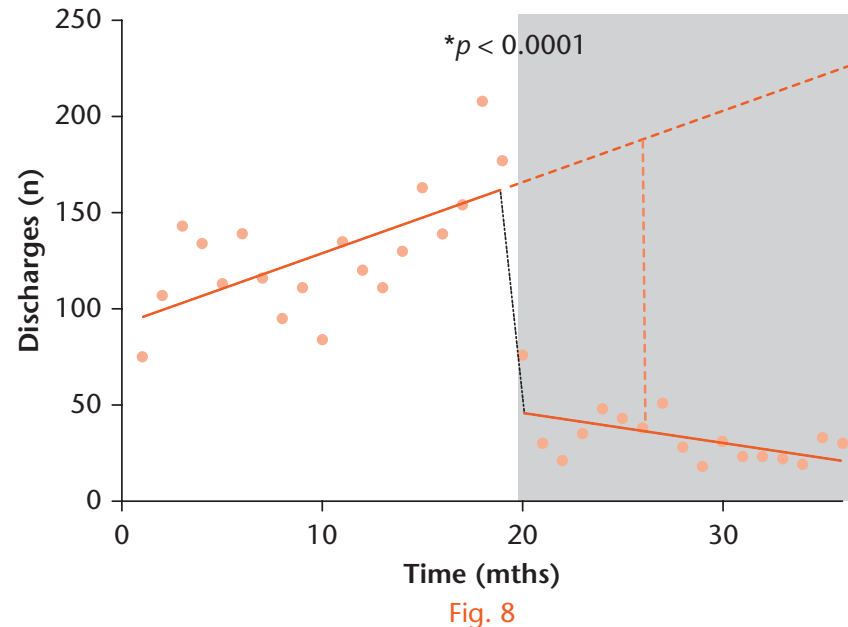

Interrupted time series (ITS) segmented regression for number of patients per month discharged directly from their first face-to-face clinic. There is a clear level and slope change. Pre-intervention slope, +3.7 ; post-intervention slope, -1.5 ; Solid dark orange line, regression line for each segment; Dashed dark orange line, counterfactual scenario; light orange dotted line, difference between counterfactual and actual scenario at six months post-intervention; Grey shading, post-intervention period.

in the counterfactual scenario (14.9 versus 59.4 ; segmented regression, $\mathrm{p}<0.0001)$.

As each missed first face-to-face appointment costs the study site $£ 160$, these results equate to an $£ 81920$ saving in the 12 months post-intervention compared with the previous 12 months, based on monthly non-attendance figures.

In terms of percentages, there was a reduction of $2.5 \%$ in non-attendance rates from first-attenders at face-toface fracture clinic from $13.1 \%$ to $10.6 \%$ (Risk Ratio (RR) $0.81,95 \%$ confidence interval $(\mathrm{Cl}) 0.71$ to 0.92 ; Odd Ratio (OR) $0.78 ; 95 \% \mathrm{Cl} 0.68$ to 0.9 ) between the preand post-intervention periods.

Figure 10 illustrates the effect of the intervention on all parameters for which ITS analysis was used. A decrease in absolute figures for non-attendance, immediate discharge and number of face-to-face clinic appointments per month can be clearly seen, in addition to decreased wait times from referral to clinic review.

Are VFCs cost-effective?: total spend by the local ccG. Calculations used to obtain the total cost to the local CCG for commissioning fracture clinic services at the study site in the financial year before and after the intervention are shown in Table III. These are based on the numbers of each clinic appointment-type and locallyagreed tariff prices (Table I). In the first year of VFC operation the study site also received the $£ 62500$ incentive bonus from the CCG for having achieved their CQUIN target; this will not be paid in subsequent years.

Pre-intervention, the CCG paid the study site $£ 1466$ 666.72 for fracture clinic services whereas postintervention it paid $£ 1336781.05$ plus the $£ 62500$ bonus (total $£ 1399$ 281.05). Thus, in their first year of operation

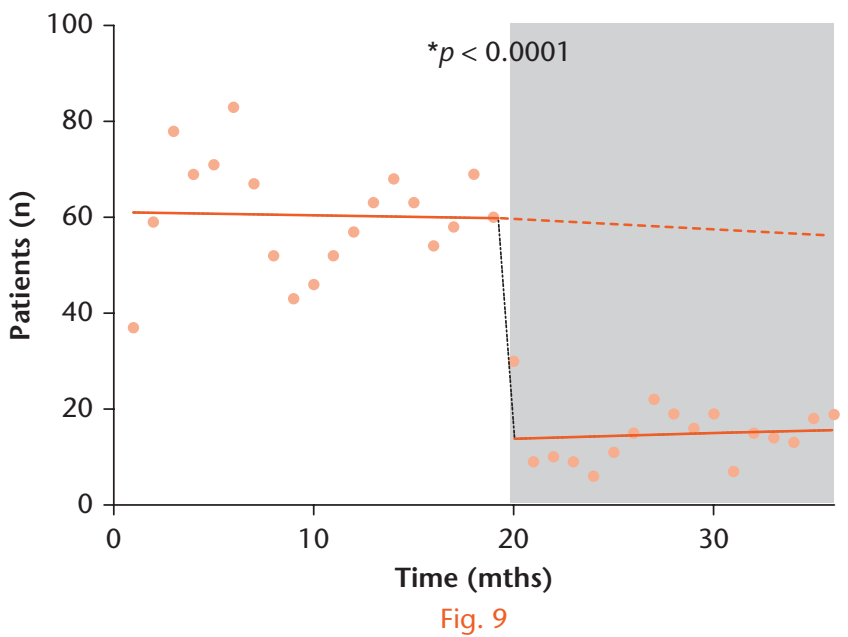

Interrupted time series (ITS) segmented regression of absolute number of non-attendances per month for face-to-face clinic. There is a clear level change. Pre-intervention slope, 0.063; post-intervention slope, 0.11; Solid dark orange line, regression line for each segment; Dashed dark orange line, counterfactual scenario; Grey shading, post-intervention period.

VFCs saved the CCG $£ 67385.67$, whilst in future years this can be expected to rise to $£ 129885.67$, assuming fracture clinic activity remains relatively constant.

The Trust's overall balance was $£ 106398.67$ less in the first year of VFCs than previously, and looks set in subsequent years to be $£ 168898.67$ less compared to pre-intervention figures due to a combination of lower CCG spending as above, provision of the VFC nurse's salary and no further CQUIN bonus payments.

\section{Discussion}

Main findings. The main findings of this study are that VFCs have led to: Fewer referrals to face-to-face fracture clinic (Figs 3 and 4), improved waiting times from referral to first orthopaedic review in clinic, ensuring more timely management decisions (Figs 5 and 6), fewer unnecessary referrals (Fig. 8) and non-attenders (Fig. 9) meaning more patients were saved the time and expense of travelling to hospital unnecessarily and saving the Trust $£ 81920$ over 12 months through reducing missed appointments, and substantial financial savings of an estimated $£ 129885.67$ per annum for the local CCG (Table III)

The null hypothesis is therefore rejected for outcome measures $a, c, d, e, f$ and $g$ but accepted for $b$ where no significant difference was detected. It can thus be postulated that this particular VFC model is both clinically and cost-effective.

Strengths and limitations. This study addresses the current 'hot topic' of VFCs, given the context of overburdened traditional fracture clinic services and recent calls by NICE and the British Orthopaedic Association for further research in this area.1,18 It is a large-scale study, including 17116 patients referred over a three 


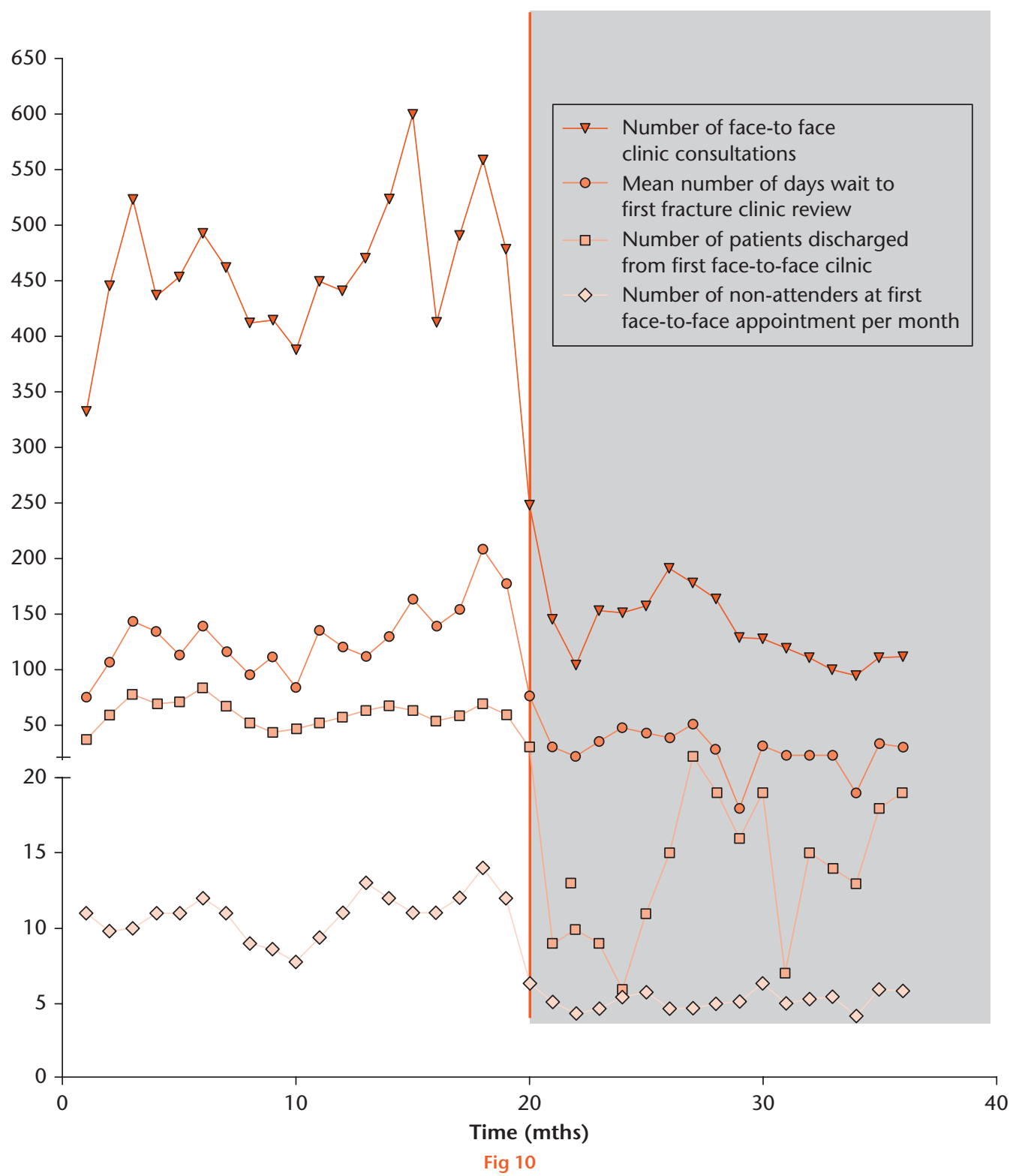

Graphical representation of the changes in key clinical performance parameters over the study period (see legend). The solid dark orange line shows the intervention (virtual fracture clinic (VFC) introduction in December 2014).

Table III. Calculations for total CCG spend for commissioning fracture clinic services at the study site for the financial years immediately pre- and post-intervention, and overall Trust balance for fracture clinic services over the same time periods

\begin{tabular}{lll}
\hline & Financial year pre-intervention & Financial year post-intervention \\
\hline Spend by CCG & & \\
New face-to-face appointments & $(5243$ at $£ 160)=£ 838880.00$ & $(1528$ at $£ 160)=£ 244480.00$ \\
Follow-up face-to-face appointments & $(6534$ at $£ 96.08)=£ 627786.72$ & $(6160$ at $£ 96.08)=£ 591852.80$ \\
Follow-up face-to-face directly from VFC & $(0$ at $£ 160)=£ 0.00$ & $(1417$ at $£ 160)=£ 226720000.00$ \\
Virtual appointments & $(0$ at $£ 64.03)=£ 0.00$ & $(4275$ at $£ 64.03)=£ 273728.25$ \\
Money for achieving CQUIN & $£ 0.00$ & $£ 62,500.00$ \\
Spend by study site & $£ 0.00$ & $£ 39013.00(£ 34530$ basic salary $+£ 4483$ London weighting) \\
VFC nurse & $x$ & $x$ \\
Administrative costs & $y$ & $y$ \\
Overhead costs (lighting, heating, telephone etc.) & $z$ & $z$ \\
Clinic nurses & $£ 1466666.72-(x+y+z)$ & $£ 1360268.05-(x+y+z)$ \\
Total Trust balance & $£ 1466666.72$ & $£ 1399281.05$ \\
Total CCG spend & $y$
\end{tabular}

CCG, Clinical Commissioning Group; VFC, virtual fracture clinic; CQUIN, Commissioning for Quality and Innovation 
year period, and is to our knowledge the only paper in England to investigate cost-effectiveness of a VFC model. It captures a whole-population group including all referral sources, thus selection bias is eliminated.

ITS is a robust quasi-experimental methodology for assessing the longitudinal effects of population-level healthcare interventions over a defined time-period, and is widely recognised as a preferred design where randomisation is unfeasible. ${ }^{22-24}$ Cochrane have recommended ITS for examining the effect of such healthcare interventions. ${ }^{27}$ Its strength lies in its ability to adjust for secular trends in pre-intervention data, biases within the data itself and in allowing easy visualisation of the intervention-effects. ${ }^{23}$ As the effect-lag after the intervention is extremely short in this case, it is possible to see stark differences in the data around the intervention point.

One shortfall of our model is that no demographic data was available to test for covariance; if it was it may have been possible to include a control group.

In relation to the literature. As of August 2016, the databases PubMed, OVID, PubMed, and Google Scholar contained only seven papers including original research, audit or commentary on the topic of virtual or telephone clinics within the NHS, ${ }^{12-17,28}$ six of these were from the Glasgow Royal Infirmary. ${ }^{12-17}$

Compared with these publications, our study is larger and examines outcomes over a longer time-period: the largest paper from Glasgow reported on 6385 patients over a one year period. ${ }^{13}$ Ours is also the only study to use a more robust methodological approach than simple before-and-after analysis. However, we have not evaluated the impact of our VFC on local ED, MIU or GPS as the Glasgow group have done; ${ }^{12,13}$ this may be an area for further research.

We found higher discharge rates from our VFC than those of previous papers, which may be a symptom of our specific VFC model. ${ }^{12,16}$ The study is generally limited in that it only applies to our specific VFC model within a particular population demographic. This is true for all published work on VFCs, however, and more publications are required in order for meta-analyses to be carried out.

Patient experience and satisfaction were not investigated as part of this study, nor were patient complaints or complications associated with the service redesign. However, upon inspecting our hospital-level data for fracture-clinic-related complaints there has been a slight reduction in these after VFC was introduced. There was only one early complaint related to VFC from all 5958 patients in the study, where the patient had concerns that no orthopaedic specialists had been involved in formulating his management plan. In line with the Glasgow group's findings there have been no identified serious untoward incidents, episodes of patient harm or medicolegal actions since the intervention. ${ }^{17}$
Implications of this study. There are potential wider societal benefits, economical and environmental advantages of reducing numbers of face-to-face clinic attendances. Each patient has their own carbon footprint, and many who attend or accompany patients must take time off work or education. Measuring the impact of this lossof-productivity, or economic and environmental costs is outside the scope of this project, but it is clear that if VFCs are adopted on a national scale there may be a significant knock-on effect.

Although we demonstrated a significant improvement in achieving the BOAST 7 target, ${ }^{5}$ we still meet this goal for only $46.4 \%$ of patients. To truly achieve this standard for all patients may require increased VFC capacity.

The high VFC discharge rate enables much-needed space to be created in face-to-face clinics for patients who need to be seen in person. The absolute reduction in patients attending face-to-face clinics has meant that clinicians potentially have more time to review each patient.

The cost-savings identified here for our local CCG represent potentially huge financial benefits nationally should VFCs be implemented more widely. However, this may present an unintended consequence whereby the Trust is financially penalised for providing this type of service, irrespective of its potential benefits in clinical parameters. Calculations from this study show that with the additional salary of the VFC nurse, the estimated loss per annum is $£ 168898.67$ after the first year. It may be, as the $B O A$ recommends in its recent position statement on VFCs, that: "safe provision of such services would not normally be feasible within the current tariff structure for a virtual review in the English NHS, and therefore special arrangements will normally need to be made with Trusts and commissioners". ${ }^{18}$

Conclusions. Our findings add valuable information to the limited literature regarding VFCs. To our knowledge this is the largest and most comprehensive study addressing clinical outcomes from VFC. The results are based on robust epidemiological methodology and are significant for a number of important parameters.

Further studies are required to investigate whether similar results are reproducible across other sites, with variation in locally agreed tariffs and VFC model. Additional evaluation of the impact of VFCs on other parameters such as patient satisfaction, adverse outcomes and time-to-surgery would also be valuable.

\section{References}

1. No authors listed. National Institute for Health and Clinical Excellence. Fractures (non-complex): assessment and management. https://www.nice.org.uk (date last accessed 23 February 2017).

2. Baker C.Accident and Emergency Statistics. House of Commons briefing paper. July 2015. www.parliament.uk/briefing-papers/sn06964.pdf (date last accessed 23 February 2017).

3. No authors listed. NHS England. A\&E Attendances and Emergency Admissions https://www.england.nhs.uk/statistics/statistical-work-areas/ae-waiting-timesand-activity/ (date last accessed February 2017). 
4. No authors listed. The Kings Fund. What's going on in A\&E? http://www.kingsfund. org.uk/projects/urgent-emergency-care/urgent-and-emergency-care-mythbusters (date last accessed February 2017).

5. No authors listed. British Orthopaedic Association. BOAST 7 fracture clinic guidelines. https://www.boa.ac.uk/wp-content/uploads/2014/12/BOAST-7.pdf (date last accessed February 2017).

6. No authors listed. The Nuffield Trust. The State of NHS Finances and the $f 22 b n$ efficiency challenge. http://www.nuffieldtrust.org.uk/our-work/projects/state-nhsfinances-and-£22bn-efficiency-challenge (date last accessed February 2017).

7. No authors listed. Health Secretary Jeremy Hunt's speech to the Kings Fund (2015). https://www.gov.uk/government/speeches/innovation-and-efficiency (date last accessed February 2017).

8. Mark DA, Fitzmaurice GJ, Haughey KA, et al. Assessment of the quality of care and financial impact of a virtual renal clinic compared with the traditional outpatient service model. Int J Clin Pract 2011;65:1100-1107.

9. Hunter J, Claridge A, James $\mathbf{S}$, et al. Improving outpatient services: the Southampton IBD virtual clinic. Postgrad Med J 2012;88:487-491.

10. Kotecha A, Bonstein K, Cable R, et al. Qualitative investigation of patients experience of a glaucoma virtual clinic in a specialist ophthalmic hospital in London, UK. BMJ Open 2015;5:e009463.

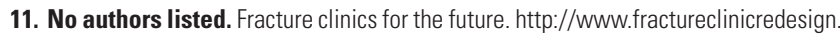
org/the-virtual-clinic/ (date last accessed February 2017).

12. Jayaram PR, Bhattacharyya R, Jenkins PJ, et al. A new "virtual" patient pathway for the management of radial head and neck fractures. J Shoulder Elbow Surg 2014;23:297-301.

13. Vardy J, Jenkins PJ, Clark $\mathbf{K}$, et al. Effect of a redesigned fracture management pathway and 'virtual' fracture clinic on ED performance. BMJ Open 2014;4:e005282.

14. Brooksbank K, Jenkins PJ, Anthony IC, et al. Functional outcome and satisfaction with a 'self-care' protocol for the management of mallet finger injuries: a case-series. J Trauma Manag Outcomes 2014;8:1.

15. Ferguson KB, McGlynn J, Jenkins P, et al. Fifth metatarsal fractures - Is routine follow-up necessary? Injury 2015;46:1664-1668

16. Jenkins PJ, Morton A, Anderson G, et al. Fracture clinic redesign reduces the cost of outpatient orthopaedic trauma care. Bone Joint Res 2016;5:33-36.

17. Jenkins PJ, Stephenson DA, Rymaszewski LA. Legal Aspects of Virtual Fracture Clinics. J Trauma Orthop. https://www.boa.ac.uk/wp-content/.../Legal-Aspects-ofVirtual-Fracture-Clinics.pdf [last accessed February 2017].

18. No authors listed. British Orthopaedic Association. Virtual Fracture Clinic Statement 23.10.15. http://www.boa.ac.uk/publications/virtual-fracture-clinicstatement-23-10-15/ (date last accessed February 2017).
19. No authors listed. NHS Data Model and Dictionary for England. http://www. datadictionary.nhs.uk/web_site_content/navigation/commissioning_data_sets_ menu.asp (date last accessed February 2017).

20. No authors listed. NHS England. Enhanced Tariff Option Spreadsheet 2015/16. https://www.england.nhs.uk/wp-content/uploads/2015/.../2015-16-eto-spreadsheet. xls (date last accessed February 2017).

21. No authors listed. NHS pay scales 2014-15. https://www.rcn.org.uk/employmentand-pay/nhs-pay-scales-2014-15 (date last accessed February 2017)

22. Bernal JL, Cummins S, Gasparrini A. Interrupted time series regression for the evaluation of public health interventions: a tutorial. Int J Epidemio/ 2016;45.

23. Wagner AK, Soumerai SB, Zhang F, Ross-Degnan D. Segmented regression analysis of interrupted time series studies in medication use research. J Clin Pharm Ther 2002;27:299-309.

24. Kontopantelis E, Doran T, Springate DA, et al. Regression based quasiexperimental approach when randomization is not an option: interrupted time series analysis. BMJ 2015:350.

25. D'Agostino RB, Belanger A, D'Agostino RBJ. A suggestion for using powerful and informative tests of normality. Am Stat 1990;44:316-321.

26. Pearson ES. Note on tests for normality. Biometrika 1931;22:423-424.

27. No authors listed. Effective Practice and Organisation of Care (EPOC). EPOC Resources for review authors 2014. https://epoc.cochrane.org/sites/epoc.cochrane. org/files/public/uploads/EPOC\%20Study\%20Designs\%20About.pdf (date last accessed February 2017)

28. Beiri A, Alani A, Ibrahim T, Taylor GJ. Trauma rapid review process: efficient outpatient fracture management. Ann R Coll Surg Eng/2006;88:408-411.

Funding Statement

None declared.

Author Contribution

A. McKirdy: Data collection and analysis, manuscript authorship (predominantly Methods / Results)

A. M. Imbuldeniya: Concept, study design, supervisor, manuscript authorship (predominantly Introduction / Discussion).

ICMJE Conflicts of Interest

None declared.

(c) 2017 McKirdy and Imbuldeniya. This is an open-access article distributed under the terms of the Creative Commons Attributions licence (CC-BY-NC), which permits unrestricted use, distribution, and reproduction in any medium, but not for commercial gain, provided the original author and source are credited. 